\title{
Carpal Tunnel Syndrome in Fabry Disease
}

\author{
Joanna Ghali • Anand Murugasu • Timothy Day • \\ Kathy Nicholls
}

Received: 19 December 2010/Revised: 25 February 2011 / Accepted: 2 March 2011 /Published online: 6 September 2011

(C) SSIEM and Springer-Verlag Berlin Heidelberg 2011

\begin{abstract}
Carpal tunnel syndrome (CTS) is a common peripheral mononeuropathy affecting up to $4 \%$ of the general population, typically women in late middle age. The incidence in patients with Fabry disease (FD) is unclear, but may affect $25 \%$ of patients with this X-linked lysosomal storage disease. We report three cases of CTS in young Caucasian male patients with classical FD, who developed CTS symptoms with supportive nerve conduction study (NCS) findings. Two patients had bilateral CTS and two had evidence of concurrent ulnar nerve neuropathy on NCS, suggesting a systemic process contributed to nerve compression. All were receiving enzyme replacement therapy (ERT) and had a moderate burden of FD complications. It is possible that an increase in connective tissue in the intracarpal canal in FD patients may be incited by injury to fibroblasts, via either accumulation of globotriaosylceramide (GL3) or local ischaemia through endothelial injury. The former hypothesis may be a more plausible explanation for the development of CTS, as histology of the flexor retinaculae from our patients has demonstrated fibroblasts with characteristic vacuolation
\end{abstract}

Communicated by: Robin Lachmann.

Competing interests: None declared.

J. Ghali $(\bowtie) \cdot$ K. Nicholls

Nephrology Department, Royal Melbourne Hospital, 1 South East

Outpatient Building, Grattan St, Parkville 3050, VIC, Australia

e-mail: joanna.ghali@monash.edu

\section{A. Murugasu}

Department of Anatomical Pathology, Royal Melbourne Hospital, Grattan St, Parkville 3050, VIC, Australia

T. Day

Department of Neurophysiology, Royal Melbourne Hospital, Grattan St, Parkville 3050, VIC, Australia and excessive myxomatous stroma, despite endothelial clearance of GL3 in these patients receiving ERT. CTS should not be overlooked in FD patients and young patients presenting with CTS should be evaluated for an underlying systemic or genetic disorder. Surgical carpal tunnel decompression was effective in our patients, already troubled by long-standing acroparesthesia, in providing sustained relief of symptoms.

\section{Introduction}

Fabry disease (FD) (OMIM 300644) is an X-linked lysosomal storage disorder due to mutations in the GLA gene, which encodes $\alpha$-galactosidase (EC 3.2.1.22), the enzyme responsible for the degradation of globotriaosylceramide (GL3). Individuals with FD develop multi-system disease via accumulation of GL3 in many tissues, including myocardium, kidney, vascular endothelium, arterial walls and connective tissue (Elleder 2003). We report three cases of carpal tunnel syndrome affecting young male patients with FD.

\section{Patient 1}

A Caucasian male, adopted as a neonate, suffered throughout his childhood with undiagnosed pain in his hands and feet, exacerbated by exercise and viral infections. FD was formally diagnosed at age 14 years, after his biological mother mother contacted the adoption agency to inform them of her own diagnosis of FD. $\alpha$-galactosidase level was reduced in peripheral blood leukocytes $(0.11 \mathrm{nmol} / \mathrm{min} / \mathrm{mg}$; normal range $0.3-2.1$ ) and in cultured fibroblasts. Subsequent genetic testing revealed an M284T mutation 
in the GLA gene. When reviewed by a nephrology service at age 20 years, his clinical features included at least 10 years of acroparesthesia, hypohidrosis, umbilical angiokeratoma and intermittent diarrhoea. He had lower limb temperature anaesthesia in a symmetrical stocking distribution to ankle level. Blood pressure, serum creatinine ( $\mathrm{Se} \mathrm{Cr}$ ) and 24 hour proteinuria were normal. Nerve conduction studies (NCS) of his lower limbs confirmed mild peripheral neuropathy. He began enzyme replacement therapy (ERT) with Agalsidase alfa (Shire HGT) at age 30 years. Four years later he developed nocturnal pain and tightness in his left thenar eminence with numbness in his left palm and lateral three digits. Nerve conduction studies were diagnostic of severe left carpal tunnel syndrome (CTS) (Table 1). $\mathrm{He}$ had low grade proteinuria (0.11-0.4 g/day), but $\mathrm{Se} \mathrm{Cr}$ and echocardiography were normal. On the Mainz severity score index (MSSI), this patient had a score of 29 (Beck 2006). Left carpal tunnel decompression relieved his symptoms. Histology of the left carpal tunnel flexor retinaculum and connective tissue from within the carpal canal identified fibrous connective tissue with scattered vacuolated cells, a small amount of myxomatous material in the stroma and PAS-positive deposits (Fig. 1). No inflammatory cells or Maltese cross lipid inclusions were identified under polarised light. Unfortunately, electron microscopy (EM) was not possible due to suboptimal specimen processing. Six years later, CTS symptoms have not recurred. Renal function remains normal and proteinuria has not progressed, but he has developed left ventricular hypertrophy with diastolic dysfunction.

\section{Patient 2}

After proband identification, this male patient was found to have a reduced WBC $\alpha$-galactosidase level at age 5 years, and the causative G128E mutation in the GLA gene was later defined. Throughout his boyhood he suffered with acroparesthesia, hypohydrosis and chronic diarrhoea. At the commencement of ERT (Agalsidase alfa) at age 34 years, glove-and-stocking sensory neuropathy affected temperature, light touch and pain sensation. Other medical problems included obstructive sleep apnoea, nasal polyps, caecal volvulus and major depression. He worked intermittently with vibratory machinery. Over the next 3 years, he developed bilateral progressive numbness and pain from hands to elbows, initially nocturnal, then during the day, especially when driving. His MSSI score was 30 . Motor examination was normal. Light touch and temperature sensation were reduced in a glove distribution in the right upper limb and in the median nerve territory in the left hand. Tinnel's sign was positive at the right wrist. NCS of his upper limbs indicated severe bilateral carpal tunnel syndrome and moderate right ulnar neuropathy at the elbow with mild sensory axonal neuropathy (Table 1). On quantitative sensory testing, the cold detection threshold was elevated but heat pain thresholds indicated hyperaesthesia, consistent with pathology of small myelinated and unmyelinated nerve fibres. Decompression of both carpal tunnels and of the right ulnar nerve relieved all symptoms. Tissue histology showed dense fibrous tissue with an increase in toluidine blue positive extracellular material. EM demonstrated lamellated zebra bodies zebra bodies within the perinuclear cytoplasm of stromal cells (Fig. 2). No cytoplasmic vacuolation of muscle, perineural tissue or vascular endothelial cells was present.

\section{Patient 3}

This Caucasian male patient also had the classical phenotype of FD (M284T mutation; belonging to the same pedigree as Patient 1). At the commencement of ERT (Agalsidase alfa) at age 41 years, FD burden included glove-and-stocking sensory neuropathy, left ventricular hypertrophy $(\mathrm{LVH})$ and proteinuria $(0.7 \mathrm{~g} / 24 \mathrm{~h})$, but radionuclide glomerular filtration rate (GFR) was normal. At the time, his MSSI score was 26. Five months later, he presented with sudden onset of severe shock-like pains in his right hand and wrist and left arm, after using heavy hand-held drilling equipment over the two preceding days, with previous occupational exposure to machinery. He recalled that similar symptoms 3-4 years earlier had settled with physiotherapy. Investigation confirmed bilateral CTS (see Table 1) and surgery provided sustained and complete symptomatic relief. Light microscopy of specimens from biopsies of the skin and flexor retinaculum of the right palm showed foamy histiocytes in the wall of the blood vessels consistent with FD.

\section{Discussion}

FD typically affects both peripheral and autonomic nerves. The mechanism of Fabry neuropathy is poorly understood, but may relate to nerve ischaemia or dysfunction from glycolipid accumulation in the vasa nervorum, or in dorsal root ganglia (Hilz et al. 2000; Schiffmann 2006). Episodic acroparesthesia in the hands and feet, occurring from childhood, affects most males and many females (Galanos et al. 2002). Patients may develop a sensory lengthdependent peripheral neuropathy, predominantly affecting small myelinated (A-delta) nerve fibres (resulting in elevated thresholds to cold stimulation) and to a lesser degree, unmyelinated $\mathrm{C}$ fibres (transmitting perception of hot stimuli) (Luciano et al. 2002; Torvin Moller et al. 
Table 1 Nerve conduction study findings and grade of carpal tunnel syndrome severity

\begin{tabular}{|c|c|c|c|c|c|c|}
\hline Patient & Nerve and site & Latency (ms) & Amplitude (mV) & Segment & $\begin{array}{l}\text { Conduction } \\
\text { velocity }(\mathrm{m} / \mathrm{s})\end{array}$ & Grade of CTS $(0-6)^{\mathrm{a}}$ \\
\hline \multirow[t]{16}{*}{ Patient 1} & MOTOR & & & & & \\
\hline & Median L & & & & & $L=4$ \\
\hline & Wrist & 5.1 & 8.6 & & & \\
\hline & Elbow & 10 & 8.5 & Wrist-elbow & 58 & \\
\hline & Ulnar $L$ & & & & & \\
\hline & Wrist & 2.2 & 11.8 & & & \\
\hline & Below elbow & 6.7 & 11.5 & Wrist-below elbow & 56 & \\
\hline & Above elbow & 8.6 & 11.5 & Below elbow-above elbow & 50 & \\
\hline & SENSORY & & & & & \\
\hline & Mixed ascending $L$ & & & & & \\
\hline & Wrist (median) & 4.4 & 26 & Elbow-wrist (median) & 69 & \\
\hline & Wrist (ulnar) & 5 & 12 & Elbow-wrist (ulnar) & 63 & \\
\hline & Digits sensory L & & & & & \\
\hline & Digit 2 (median) & Absent & & & & \\
\hline & Digit 1 (median) & 3.1 & 2 & Wrist-digit 1 (median) & 37 & \\
\hline & Digit 5 (ulnar) & 2.3 & 8 & Wrist-digit 5 (ulnar) & 62 & \\
\hline \multirow[t]{20}{*}{ Patient 2} & MOTOR & & & & & \\
\hline & Median $R$ & & & & & $R=4$ \\
\hline & Wrist & 5.6 & 5.6 & & & \\
\hline & Elbow & 11.5 & 5.7 & Wrist-elbow & 51 & \\
\hline & Ulnar $R$ & & & & & \\
\hline & Wrist & 2.5 & 7.4 & & & \\
\hline & Below elbow & 6.5 & 6.4 & Wrist-below elbow & 53 & \\
\hline & Above elbow & 10.3 & 6.3 & Below elbow-above elbow & 32 & \\
\hline & Median $L$ & & & & & $L=4$ \\
\hline & Wrist & 6.1 & 6 & & & \\
\hline & Ulnar $L$ & & & & & \\
\hline & Wrist & 2.7 & 9.9 & & & \\
\hline & SENSORY & & & & & \\
\hline & Mixed ascending $R$ & & & & & \\
\hline & Wrist (median) & 5.4 & 28 & Elbow-wrist (median) & 62 & \\
\hline & Wrist (ulnar) & 6.6 & 10 & Elbow-wrist (ulnar) & 48 & \\
\hline & Digits sensory $R$ & & & & & \\
\hline & Digit 2 (median) & Absent & & & & \\
\hline & Digit 1 (median) & Absent & & & & \\
\hline & Digit 5 (ulnar) & 2.7 & 2 & Wrist-digit 5 (ulnar) & 52 & \\
\hline \multirow[t]{11}{*}{ Patient 3} & MOTOR & & & & & \\
\hline & Median $R$ & & & & & $R=3$ \\
\hline & Wrist & 5.3 & 9.5 & & & \\
\hline & Elbow & 11.55 & 9.1 & Wrist-elbow & 52.8 & \\
\hline & Ulnar $R$ & & & & & \\
\hline & Wrist & 3.15 & 10.4 & & & \\
\hline & Below elbow & 4.85 & 10.1 & Wrist-below elbow & 47.1 & \\
\hline & Above elbow & 10.55 & 10 & Below elbow-above elbow & 43.9 & \\
\hline & Median $L$ & & & & & $L=3$ \\
\hline & Wrist & 4.6 & 9 & & & \\
\hline & Elbow & 11.4 & 7.3 & Wrist-elbow & 48.5 & \\
\hline
\end{tabular}


Table 1 (continued)

\begin{tabular}{|c|c|c|c|c|c|c|}
\hline Patient & Nerve and site & Latency (ms) & Amplitude (mV) & Segment & $\begin{array}{l}\text { Conduction } \\
\text { velocity }(\mathrm{m} / \mathrm{s})\end{array}$ & Grade of CTS $(0-6)^{\mathrm{a}}$ \\
\hline & \multicolumn{6}{|l|}{ Ulnar $L$} \\
\hline & Wrist & 2.65 & 9.9 & & & \\
\hline & Below elbow & 4.55 & 9.5 & Wrist-below elbow & 52.6 & \\
\hline & Above elbow & 9.7 & 9.1 & Below elbow-above elbow & 48.5 & \\
\hline & \multicolumn{6}{|l|}{ SENSORY } \\
\hline & \multicolumn{6}{|l|}{ Mixed ascending $R$} \\
\hline & Wrist (median) & 2 & 10.4 & Elbow-wrist (median) & 40 & \\
\hline & Wrist (ulnar) & 1.15 & 7.8 & Elbow-wrist (ulnar) & 62.2 & \\
\hline & \multicolumn{6}{|l|}{ Digits sensory $R$} \\
\hline & Digit 2 (median) & 3.05 & 1.6 & Wrist-digit 2 (median) & 44.3 & \\
\hline & Digit 1 (median) & 2.85 & 5.5 & Wrist-digit 1 (median) & 35.1 & \\
\hline & Digit 5 (ulnar) & 2 & 4.5 & Wrist-digit 5 (ulnar) & 55 & \\
\hline & \multicolumn{6}{|l|}{ Mixed ascending $L$} \\
\hline & Wrist (median) & 1.9 & 21 & Elbow-wrist (median) & 42.1 & \\
\hline & Wrist (ulnar) & 1.35 & 11 & Elbow-wrist (ulnar) & 59.3 & \\
\hline & \multicolumn{6}{|l|}{ Digits sensory $L$} \\
\hline & Digit 2 (median) & 3.2 & 3.8 & Wrist-digit 2 (median) & 40.6 & \\
\hline & Digit 1 (median) & 2.8 & 3.4 & Wrist-digit 1 (median) & 35.7 & \\
\hline & Digit 5 (ulnar) & 2.1 & 2.9 & Wrist-digit 5 (ulnar) & 54.3 & \\
\hline
\end{tabular}

CTS carpal tunnel syndrome, $L$ left, $R$ right

"As defined by the "Canterbury Scale" (Bland 2000)

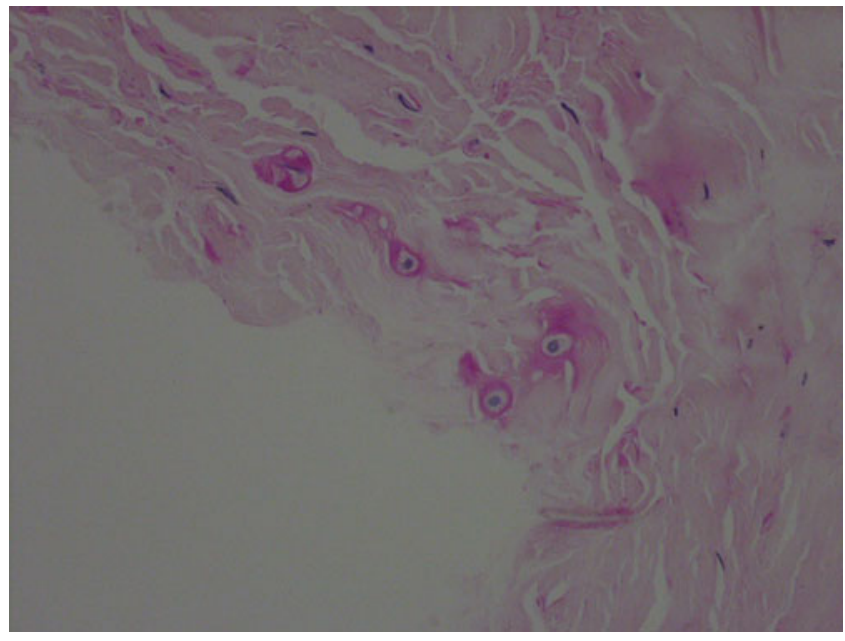

Fig. 1 Light microscopy image showing connective tissue with vacuolated fibroblasts, biopsied from the carpal tunnel of Patient 1

2009). ERT improves small fibre function (Hilz et al. 2004). Light and electron microscopy of peripheral nerve specimens has shown lipid granules within the endothelium of epineural arterioles, perineural fibroblasts, smooth muscle cells and pericytes, but inclusions in Schwann cells, unmyelinated and myelinated axons are less consistent findings (Kocen and Thomas 1970; Sima and Robertson 1978; Kaye et al. 1988; Toyooka and Said 1997).

CTS, a mononeuropathy of the median nerve, was superimposed on these classic FD manifestations in the three patients described. In the general population, CTS is proposed to result from increased pressure within the carpal tunnel, with subsequent median nerve ischaemia, epineural oedema and fibroblast invasion, culminating in the development of constrictive scar tissue around the nerve (Gelberman et al. 1981; Werner and Andary 2002; Uchiyama et al. 2010). While CTS is associated with a number of systemic diseases and some mechanical and occupational factors (Mattioli et al. 2009; Uchiyama et al. 2010), a genetic predisposition for CTS has also been shown (Hakim et al. 2002; Lozano-Calderon et al. 2008). CTS presenting in childhood should prompt consideration of an underlying lysosomal storage disorder or other genetic condition (Van Meir and De Smet 2003; Sri-Ram et al. 2007; Yuen et al. 2007). Patients presenting to Australian general practice with symptoms of CTS are predominantly female (male-female ratio $2: 3$ ) and $46 \%$ are aged 45-65 years (Charles et al. 2009). A similar gender ratio is reported in a Swedish general population cohort, with a population prevalence of "clinically certain" CTS at 4\% overall and 2\% in males (Atroshi et al. 1999). 


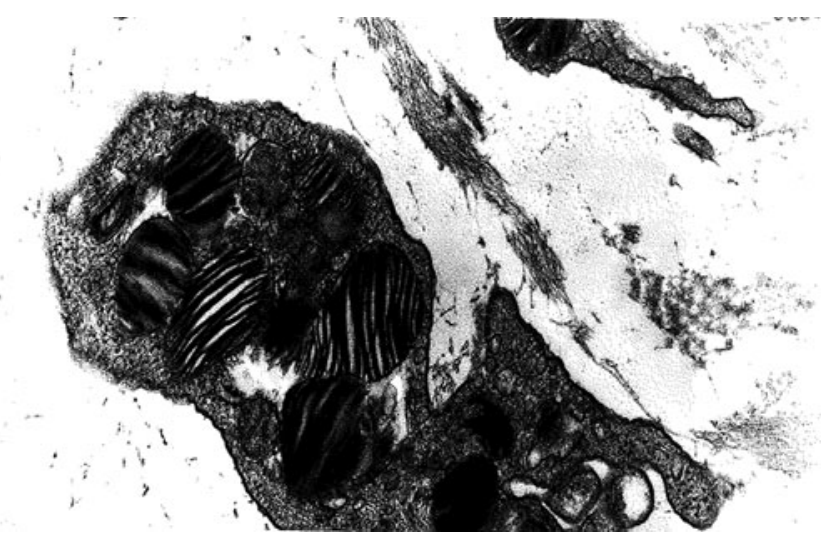

Fig. 2 EM photograph $(20,000 \times)$ of "zebra bodies" within a fibroblast from tissue taken from the carpal tunnel at the time of surgical decompression from Patient 2

In patients with $\mathrm{FD}$, the prevalence of CTS is unclear but appears to be higher than in the general population, independent of the confounding association between FD and renal failure. In a study of 22 consecutive patients with FD aged 20-52 years with creatinine clearance $>50 \mathrm{~mL} / \mathrm{min}$, six $(27 \%)$ had median nerve entrapment at the wrist, as identified by delayed sensory conduction. However, while these patients suffered hand acroparesthesia, they had no clinical features of CTS (Luciano et al. 2002). Another study evaluating peripheral nerve involvement of 12 female FD patients found 3 women (25\%), aged between 43 and 60 years, had clinical symptoms of CTS, with confirmatory NCS (Laaksonen et al. 2008).

The 3 male patients described received medical care within a state-wide cohort of 30 male FD patients. Based on their MSSI scores, they had a moderate disease burden at the time of developing CTS. The cohort of 30 male FD patients includes 8 deceased males (all of whom received ERT), who scored a median MSSI score of 36 (range 23-50) at the time of their death. The disease burden of the 3 males with CTS is similar to the remainder of the living FD cohort (which includes 16 patients receiving ERT and 2 participating in a trial of oral chaperone therapy), who scored a median of 28 (range 3-49) on the MSSI. Patients were only investigated for CTS if they had suggestive symptoms; no other individuals in this cohort have had NCS of their upper limbs. The FD patients with CTS developed this condition in their $30 \mathrm{~s}$, younger than the typical age of presentation in the general population. Patients 2 and 3 had possible environmental triggers and patient 3 had performed heavy repetitive manual work with vibrating equipment within $24 \mathrm{~h}$ of his acute presentation. Although both of these men presented with CTS after receiving ERT for many months, they recalled prior milder symptoms consistent with CTS. Patients 1 and 2 also had evidence of ulnar nerve neuropathy on NCS and Patient
2 required a right ulnar nerve decompression, suggesting that systemic rather than solely local processes were involved in the evolution of nerve compression.

CTS evolved in our patients despite ERT. Histology of the excised flexor retinaculae and perineural tissue demonstrated vacuolation of fibroblasts and an increase in extracellular material. Although nerve biopsies were not performed, rapid improvement in symptoms upon surgical decompression suggests a compressive aetiology for CTS rather than direct neuronal injury. Generally, the endothelium from our carpal tunnel biopsies on patients on long-term ERT was clear of GL3 inclusions, although GL3 storage persisted in tissue fibroblasts. This is consistent with other studies of long-term ERT for FD, where persistent storage of GL3 has been demonstrated in vascular smooth muscle, stromal fibroblasts, pericytes and skeletal muscle fibres (Keslova-Veselikova et al. 2008) and subtotal removal demonstrated in cultured skin fibroblasts (Askari et al. 2007). GL3 clearance varies between different cell types (Murray et al. 2007). Renal, myocardial and dermal endothelium clears after 5-6 months of ERT, and sustained clearance from vascular endothelium has been demonstrated after 30-36 months of ERT (Eng et al. 2001; Wilcox et al. 2004; Keslova-Veselikova et al. 2008; Thurberg et al. 2009). Persistence of inclusions within fibroblasts, despite in vitro evidence that Agalsidase alfa and beta are internalised by fibroblasts with effective lysosomal GL3 clearance (Keslova-Veselikova et al. 2008), may be explained through low expression of cation-independent mannose-6-phosphate (M6P) receptors, as has been demonstrated in Fabry Schwann cells (Kawashima et al. 2007). A different and less effective mechanism of enzyme uptake by fibroblasts compared to endothelial cells has also been hypothesised (Mayes et al. 1982; Hasholt et al. 1988). Endothelial cells may effectively clear GL3 because ERT is delivered at high concentration directly to the endothelium through intravenous administration, which may also protect new endothelium against GL3 accumulation. Therefore, it is plausible that despite ERT, ongoing GL3 accumulation in fibroblasts within the carpal tunnel may continue to incite a response resulting in increased extracellular matrix, culminating in the clinical picture of CTS. The relative absence of endothelial cell inclusions in our patients on ERT suggests that the alternative hypothesis - that endothelial involvement contributes to local ischaemia in the carpal tunnel - is less likely.

\section{Conclusion}

CTS may be an under-recognised complication of FD. In this series, three male patients with classical FD (representing $10 \%$ of our FD cohort), developed CTS at 
a younger age than expected for the general population, despite receiving ERT. The symptomatic benefit of carpal tunnel decompression in these patients, already troubled by long-standing acroparesthesia and sensory neuropathy, highlights the need for appropriate recognition and management of CTS in FD. Histology identified increased extracellular matrix material around collagen fibres as well as inclusions of GL3 in retinacular fibroblasts. An underlying genetic disorder should be considered in the case of children or young adults who develop CTS.

\section{Synopsis}

Carpal tunnel syndrome may be a sequela of classical Fabry Disease (through injury to fibroblasts or local ischaemia within the carpal tunnel) despite long-term enzyme replacement therapy, and effective relief of symptoms can be achieved with surgical decompression.

\section{References}

Askari H, Kaneski CR, Semino-Mora C, Desai P, Ang A, Kleiner DE, Perlee LT, Quezado M, Spollen LE, Wustman BA, Schiffmann R (2007) Cellular and tissue localization of globotriaosylceramide in Fabry disease. Virchows Arch 451(4):823-834

Atroshi I, Gummesson C, Johnsson R, Ornstein E, Ranstam J, Rosen I (1999) Prevalence of carpal tunnel syndrome in a general population. JAMA 282(2):153-158

Beck M (2006) The Mainz Severity Score Index (MSSI): development and validation of a system for scoring the signs and symptoms of Fabry disease. Acta Paediatr Suppl 95(451):43-46

Bland JD (2000) A neurophysiological grading scale for carpal tunnel syndrome. Muscle Nerve 23(8):1280-1283

Charles J, Fahridin S, Britt H (2009) Carpal tunnel syndrome. Aust Fam Physician 38(9):665

Elleder M (2003) Sequelae of storage in Fabry disease - pathology and comparison with other lysosomal storage diseases. Acta Paediatr Suppl 92(443):46-53, discussion 45

Eng CM, Guffon N, Wilcox WR, Germain DP, Lee P, Waldek S, Caplan L, Linthorst GE, Desnick RJ (2001) Safety and efficacy of recombinant human alpha-galactosidase A-replacement therapy in Fabry's disease. N Engl J Med 345(1):9-16

Galanos J, Nicholls K, Grigg L, Kiers L, Crawford A, Becker G (2002) Clinical features of Fabry's disease in Australian patients. Intern Med J 32(12):575-584

Gelberman RH, Hergenroeder PT, Hargens AR, Lundborg GN, Akeson WH (1981) The carpal tunnel syndrome. A study of carpal canal pressures. J Bone Joint Surg Am 63(3):380-383

Hakim AJ, Cherkas L, El Zayat S, MacGregor AJ, Spector TD (2002) The genetic contribution to carpal tunnel syndrome in women: a twin study. Arthritis Rheum 47(3):275-279

Hasholt L, Wandall A, Sorensen SA (1988) Enzyme replacement in Fabry endothelial cells and fibroblasts: uptake experiments and electron microscopical studies. Clin Genet 33(5):360-371

Hilz MJ, Stemper B, Kolodny EH (2000) Lower limb cold exposure induces pain and prolonged small fiber dysfunction in Fabry patients. Pain $84(2-3): 361-365$
Hilz MJ, Brys M, Marthol H, Stemper B, Dutsch M (2004) Enzyme replacement therapy improves function of $\mathrm{C}-$, Adelta-, and Abeta-nerve fibers in Fabry neuropathy. Neurology 62(7): $1066-1072$

Kawashima I, Watabe K, Tajima Y, Fukushige T, Kanzaki T, Kanekura T, Sugawara K, Ohyanagi N, Suzuki T, Togawa T, Sakuraba H (2007) Establishment of immortalized Schwann cells from Fabry mice and their low uptake of recombinant alpha-galactosidase. J Hum Genet 52(12):1018-1025

Kaye EM, Kolodny EH, Logigian EL, Ullman MD (1988) Nervous system involvement in Fabry's disease: clinicopathological and biochemical correlation. Ann Neurol 23(5):505-509

Keslova-Veselikova J, Hulkova H, Dobrovolny R, Asfaw B, Poupetova H, Berna L, Sikora J, Golan L, Ledvinova J, Elleder M (2008) Replacement of alpha-galactosidase A in Fabry disease: effect on fibroblast cultures compared with biopsied tissues of treated patients. Virchows Arch 452(6):651-665

Kocen RS, Thomas PK (1970) Peripheral nerve involvement in Fabry's disease. Arch Neurol 22(1):81-88

Laaksonen SM, Roytta M, Jaaskelainen SK, Kantola I, Penttinen M, Falck B (2008) Neuropathic symptoms and findings in women with Fabry disease. Clin Neurophysiol 119(6):1365-1372

Lozano-Calderon S, Anthony S, Ring D (2008) The quality and strength of evidence for etiology: example of carpal tunnel syndrome. J Hand Surg Am 33(4):525-538

Luciano CA, Russell JW, Banerjee TK, Quirk JM, Scott LJ, Dambrosia JM, Barton NW, Schiffmann R (2002) Physiological characterization of neuropathy in Fabry's disease. Muscle Nerve 26(5):622-629

Mattioli S, Baldasseroni A, Bovenzi M, Curti S, Cooke RM, Campo G, Barbieri PG, Ghersi R, Broccoli M, Cancellieri MP, Colao AM, Dell'omo M, Fateh-Moghadam P, Franceschini F, Fucksia S, Galli P, Gobba F, Lucchini R, Mandes A, Marras T, Sgarrella C, Borghesi S, Fierro M, Zanardi F, Mancini G, Violante FS (2009) Risk factors for operated carpal tunnel syndrome: a multicenter population-based case-control study. BMC Public Health 9:343

Mayes JS, Cray EL, Dell VA, Scheerer JB, Sifers RN (1982) Endocytosis of lysosomal alpha-galactosidase A by cultured fibroblasts from patients with Fabry disease. Am J Hum Genet 34(4):602-610

Murray GJ, Anver MR, Kennedy MA, Quirk JM, Schiffmann R (2007) Cellular and tissue distribution of intravenously administered agalsidase alfa. Mol Genet Metab 90(3):307-312

Schiffmann R (2006) Neuropathy and Fabry disease: pathogenesis and enzyme replacement therapy. Acta Neurol Belg 106(2):61-65

Sima AA, Robertson DM (1978) Involvement of peripheral nerve and muscle in Fabry's disease. Histologic, ultrastructural, and morphometric studies. Arch Neurol 35(5):291-301

Sri-Ram K, Vellodi A, Pitt M, Eastwood DM (2007) Carpal tunnel syndrome in lysosomal storage disorders: simple decompression or external neurolysis? J Pediatr Orthop B 16(3):225-228

Thurberg BL, Fallon JT, Mitchell R, Aretz T, Gordon RE, O'Callaghan MW (2009) Cardiac microvascular pathology in Fabry disease: evaluation of endomyocardial biopsies before and after enzyme replacement therapy. Circulation 119(19):2561-2567

Torvin Moller A, Winther Bach F, Feldt-Rasmussen U, Rasmussen A, Hasholt L, Lan H, Sommer C, Kolvraa S, Ballegaard M, Staehelin Jensen T (2009) Functional and structural nerve fiber findings in heterozygote patients with Fabry disease. Pain 145 (1-2):237-245

Toyooka K, Said G (1997) Nerve biopsy findings in hemizygous and heterozygous patients with Fabry's disease. J Neurol 244(7): 464-468 
Uchiyama S, Itsubo T, Nakamura K, Kato H, Yasutomi T, Momose T (2010) Current concepts of carpal tunnel syndrome: pathophysiology, treatment, and evaluation. J Orthop Sci 15(1):1-13

Van Meir N, De Smet L (2003) Carpal tunnel syndrome in children. Acta Orthop Belg 69(5):387-395

Werner RA, Andary M (2002) Carpal tunnel syndrome: pathophysiology and clinical neurophysiology. Clin Neurophysiol 113(9):1373-1381
Wilcox WR, Banikazemi M, Guffon N, Waldek S, Lee P, Linthorst GE, Desnick RJ, Germain DP (2004) Long-term safety and efficacy of enzyme replacement therapy for Fabry disease. Am J Hum Genet 75(1):65-74

Yuen A, Dowling G, Johnstone B, Kornberg A, Coombs C (2007) Carpal tunnel syndrome in children with mucopolysaccaridoses. J Child Neurol 22(3):260-263 